\section{Combining against tuberculosis}

\section{By Lev Osherovich, Senior Writer}

Although a handful of new therapies are in the pipeline to treat tuberculosis, it is unlikely that any single molecule will suffice to treat the disease because of the rapid emergence of drug-resistant strains. Drawing on lessons learned from the fight against HIV, the nonprofit Global Alliance for TB Drug Development has partnered with the Critical Path Institute to map out a strategy for testing combinations of multiple classes of therapeutics in industry's pipeline.

The partnership, called The Critical Path to TB Drug Regimens, hopes to bring companies and regulators together to establish standards for testing combination therapies. The initiative is receiving financial and organizational support from the Bill \& Melinda Gates Foundation and the World Health Organization, and it plans to work with 10 companies that have preclinical and clinical TB programs: Johnson \& Johnson, sanofi-aventis Group, Pfizer Inc., AstraZeneca plc, Bayer AG, GlaxoSmithKline plc, Otsuka Pharmaceutical Co. Ltd., Novartis AG, Sequella Inc. and Anacor Pharmaceuticals Inc.

Current TB therapies involve long courses of treatment with a quartet of old antibiotics. However, drug-resistant strains and poor patient compliance have blunted the effectiveness of the old drugs.

"TB is an archetypical example of a disease that must be treated with combination therapy," said Mel Spigelman, president and CEO of the Global Alliance for TB Drug Development (TB Alliance).

Spigelman said that when the TB Alliance began developing drug candidates in 2000, there were few options available for new combination therapies. Now, a decade of progress by pharma and biotech companies has yielded about 10 new therapeutics, so the time has come to figure out the most potent combinations, he said (see Table 1, “Tuberculosis pipeline”).

Individual companies "can get a compound to the point where it can be looked at as a drug, but once you get into the clinical space, you need to think about a regimen," said Spigelman. "That's when you have to bring all the companies into the same boat."

The TB Alliance has paved the way with preclinical studies of various combinations of about 10 compounds in development, Spigelman said. Identifying the strongest pairings and regimens among the new candidates has taken two years of preclinical work.

He declined to disclose which compounds have been tested and said it was up to the companies to individually make those announcements.

On the heels of the preclinical results, the TB Alliance now plans to bring companies together to run clinical trials and pool data about
Table 1. Tuberculosis pipeline. Clinical-stage compounds for treating tuberculosis.

Source: BCIQ: BioCentury Online Intelligence; Working Group on New TB Drugs

\begin{tabular}{lll}
\hline Compound & Sponsors & Phase \\
\hline Gatifloxacin & Institute of Research for Development & Phase III \\
\hline Moxifloxacin & $\begin{array}{l}\text { Bayer AG (Xetra:BAY)/Global Alliance for TB Drug } \\
\text { Development (TB Alliance) }\end{array}$ & Phase III \\
\hline PA-824 & Novartis AG (NYSE:NVS; SIX:NOVN)/TB Alliance & Phase II \\
\hline TMC207 & Johnson \& Johnson (NYSE:JNJ)/TB Alliance & Phase II \\
\hline OPC-67683 & Otsuka Pharmaceutical Co. Ltd. (Tokyo:4768) & Phase II \\
\hline LL 3858 & Lupin Ltd. (NSE:LUPIN; BSE:500257) & Phase II \\
\hline Linezolid & Pfizer Inc. (NYSE:PFE) & Phase II \\
\hline Rifapentine & sanofi-aventis Group (Euronext:SAN; NYSE:SNY) & Phase II \\
\hline AZD5847 & AstraZeneca plc (LSE:AZN; NYSE:AZN) & Phase I \\
\hline SQ109 & Sequella Inc. & Phase I \\
\hline PF-2341272 & Pfizer & Phase I
\end{tabular}

which combinations work most effectively against Mycobacterium tuberculosis in humans.

"We've identified regimens that look like they're better in the mouse than anything used alone today" or any of the new compounds by themselves, said Spigelman. "At the most optimistic, we could have something in human testing by the end of 2010."

Because compounds in the pipeline hit a range of previously untargeted bacterial proteins, M. tuberculosis strains that are resistant to current therapies are expected to be susceptible to the new molecules.

Spigelman noted that combination therapy is essential to prevent the emergence of strains resistant to the new drugs.

"TB drug resistance is a man-made phenomenon-the bugs don't develop resistance until they've been exposed to drugs," he said.

Spigelman would not disclose which drug combinations would be tried first but said that early programs would involve "drugs already in the clinic together with drugs that are representative of new classes."

\section{Resisting resistance}

In addition to bringing drug developers together, the other objective of the program is to urge regulators to accept new endpoints in TB trials. The Critical Path Institute (C-Path), which was formed in 2005 with the mission of paving the regulatory road for new therapies in a range of key areas, will seek consensus among companies and the FDA on how to run and interpret clinical trials of TB drug combinations.

"The prior path from the FDA is to develop a drug, test it, then combine it" with other drugs, said Raymond Woosley, president and CEO of C-Path. "This requires many years of work," by which time drug resistance has had time to emerge.

"To eradicate TB, just as with HIV, you really need combinations" from the start, he told SciBX.

Woosley hopes to persuade regulators to allow drug combinations to advance into large-scale clinical testing based on data from preclinical and short-term studies in humans, using new biomarkers 
and imaging data as a proxy for conventional efficacy endpoints.

C-Path serves as "a neutral ground where companies, regulators and academics can come together" to formulate clinical and regulatory strategies, said Woosley. While the TB Alliance does the preclinical footwork, he said, C-Path will focus on identifying the human biomarkers for combination trials.

The partnership also has been working with companies and regulators to develop "an agreement in advance on what methods will be useful and informative," said Woosley. "We need something like a CD4 count," which is the measure of T cell survival that serves as a proxy for HIV drug efficacy.

Bringing everyone together will likely involve the FDA at an earlier than usual stage in drug development, said Woosley.

"Companies have always met with the FDA on their drug development process, but it's always about their drug" alone rather than in combination, he noted.

Woosley said he hopes to get companies and regulators together midyear for a discussion about trial design and "a legal agreement getting all of the companies comfortable sharing data."

One example of how such a partnership between regulators and companies can lead to an optimized trial design that simultaneously tests multiple drug candidates is the I-SPY 2 trial. The study was launched last month and integrates biomarkers, adaptive trial designs and bioinformatics to speed the identification of compound combinations for breast cancer. ${ }^{1}$

The next step for the TB partnership is to recruit trial sites with at-risk populations, primarily in the developing world. According to Woosley, the Gates Foundation will help enroll patients through its existing network of trial centers.
Spigelman said the TB Alliance has reached out to candidate trial sites from previous monotherapy trials in South Africa, where TB is a significant public health threat. He added that he is working with the Centers for Disease Control and Prevention and the NIH to identify additional sites.

Woosley and Spigelman both noted that TB is caused by multiple strains of $M$. tuberculosis and that different latent forms prevail in different parts of the world. As a result, they said, it's likely that several different drug combinations or regimens need to be developed concurrently.

Osherovich, L. SciBX 3(14); doi:10.1038/scibx.2010.424

Published online April 8, 2010

\section{REFERENCES}

1. Usdin, S. BioCentury 13(18), A7-A10; March 22, 2010

\section{COMPANIES AND INSTITUTIONS MENTIONED}

Anacor Pharmaceuticals Inc., Palo Alto, Calif. AstraZeneca plc (LSE:AZN; NYSE:AZN), London, U.K. Bayer AG (Xetra:BAY), Leverkusen, Germany Bill \& Melinda Gates Foundation, Seattle, Wash. Centers for Disease Control and Prevention, Atlanta, Ga. Critical Path Institute, Tucson, Ariz.

Food and Drug Administration, Silver Spring, Md. GlaxoSmithKline plc (LSE:GSK; NYSE:GSK), London, U.K. Global Alliance for TB Drug Development, New York, N.Y. Johnson \& Johnson (NYSE:JNJ), New Brunswick, N.J. National Institutes of Health, Bethesda, Md.

Novartis AG (NYSE:NVS; SIX:NOVN), Basel, Switzerland Otsuka Pharmaceutical Co. Ltd. (Tokyo:4768), Tokyo, Japan Pfizer Inc. (NYSE:PFE), New York, N.Y. sanofi-aventis Group (Euronext:SAN; NYSE:SNY), Paris, France Sequella Inc., Rockville, Md.

World Health Organization, Geneva, Switzerland 